\title{
Quantitative measurements of heat transfer coefficient of impinging round jets at high Reynolds number on a perpendicular and inclined heated plate.
}

\author{
by Ph. Planquart*, M. Delsipee*, M. Dubois** and JM Buchlin* \\ *von Karman Institute, Ch. de Waterloo 72, 1640 Rhode-Saint-Genèse, Belgium, philippe.planquart@vki.ac.be \\ **John Cockerill Industry, Av. Grenier, 1, 4100 Seraing, Belgium, michel.dubois@johncockerill.com
}

\section{Abstract}

Impinging jets are extensively used in the steel industry for high speed cooling of metal sheet. They can provide a high controlled heat transfer coefficient, which is mandatory for fast cooling applications, like the annealing of metal belts.

Values of heat transfer coefficient can be found in the literature for standard conditions [1], but for very high Reynolds number (above 100.000), the data are rather sparse and the standard correlations are not valid anymore. Furthermore, in the literature, most of the results are published for impinging jets perpendicular to the substrate. The impinging jets can be round nozzles or slot nozzles depending on the application. In case of multiple jets, we can have either an Array of Round Nozzles (ARN) or an Array of Slot Nozzles (ASN).

The paper presents a quantitative infrared thermography investigation of the heat transfer obtained with an ARN configuration, consisting of 1 central jet surrounded by six additional as sketched and visualized in Figure 1. The arrangement has the following characteristics:

Diameter of the nozzle: $D=14 \mathrm{~mm}$,

Standoff distance: $Z=50,70 \& 85 \mathrm{~mm}$

Nozzle spacing: $W=70 \mathrm{~mm}$.
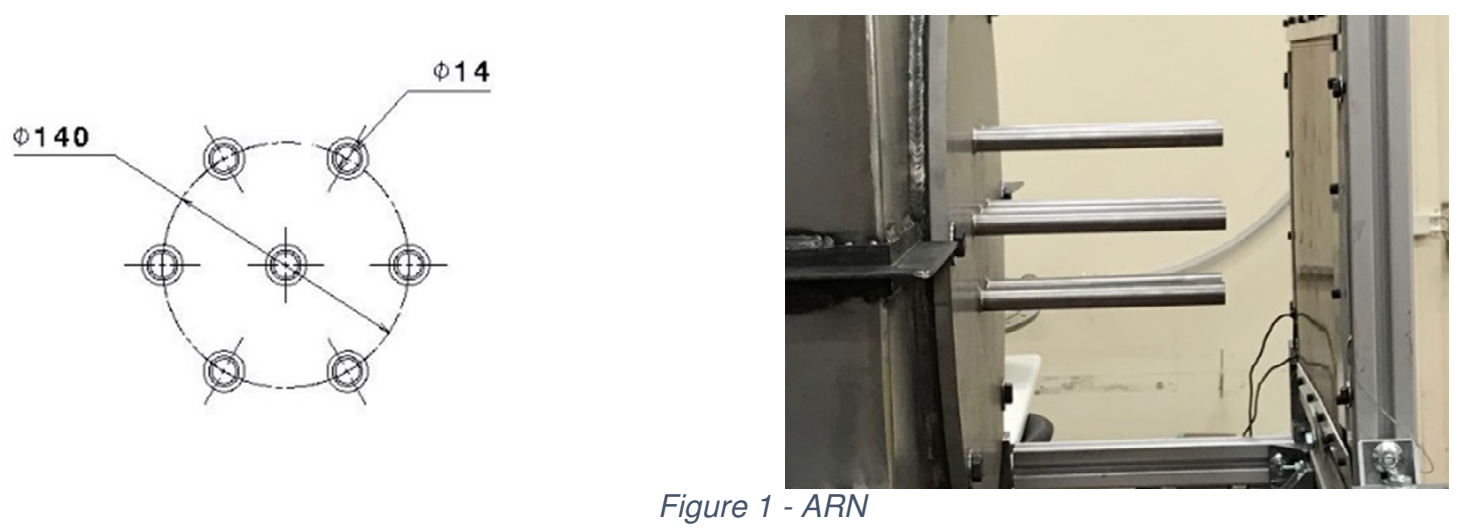

The flat plate is uniformly heated by Joule effect; its temperature is measured by an infrared camera. The thermogrammes are processes to determine the mapping of the heat transfer coefficient knowing the imposed heat flux and the jet temperature. The infrared radiometer is a FLIR 6555sc thermocam with an announced NETD of less than $30 \mathrm{mK}$. The camera provides images of $640 * 480$ pixels.

Test have been performed for a Reynolds number ranging from 40.000 to 160.000 . Three different configurations have been tested: A standard configuration perpendicular to the target with a normalised standoff distance $Z / D$ varying from 3.5 to 6 . A second configuration with the same jet arrangement but with a $10^{\circ}$ inclined plate. In this configuration, the $Z / D$ value is changing for the different jets. The last configuration consists of a $10^{\circ}$ inclined plate but with constant $Z / D$ for the different jets. 
The three configurations are shown in Figure 2 with a typical heat transfer map obtained for a Reynolds number of approximatively 80.000. Corresponding temperature profiles along the centreline are also plotted.

In the perpendicular configuration, we obtained a symmetric map of heat transfer coefficient. The heat transfer value is extracted from a hexagon geometry centred on the geometry impact point of the central jet. Values of mean heat transfer coefficient are obtained as function of the Reynolds number.

With the inclined plate configuration, we measured different values of heat transfer coefficient depending on the Z/D value of the local jets. The dissymmetry is clearly visible on the heat transfer coefficient map. Mean value inside the hexagon surrounding the central jet are extracted and compared with the perpendicular configuration.

In the last configuration, all the seven different jets produce similar maximum heat transfer coefficients, but we observe a dissymmetry in the shape of the heat transfer map. The effect of the plate inclination can be seen on the imprint of the central jet.

The cooling performance is evaluated on the basis of the mean heat transfer coefficient calculated on the representative cell of the configuration (hexagon centred on the central jet). Results for the different Reynolds numbers and different configurations will be presented in the final paper.

\section{Perpendicular}
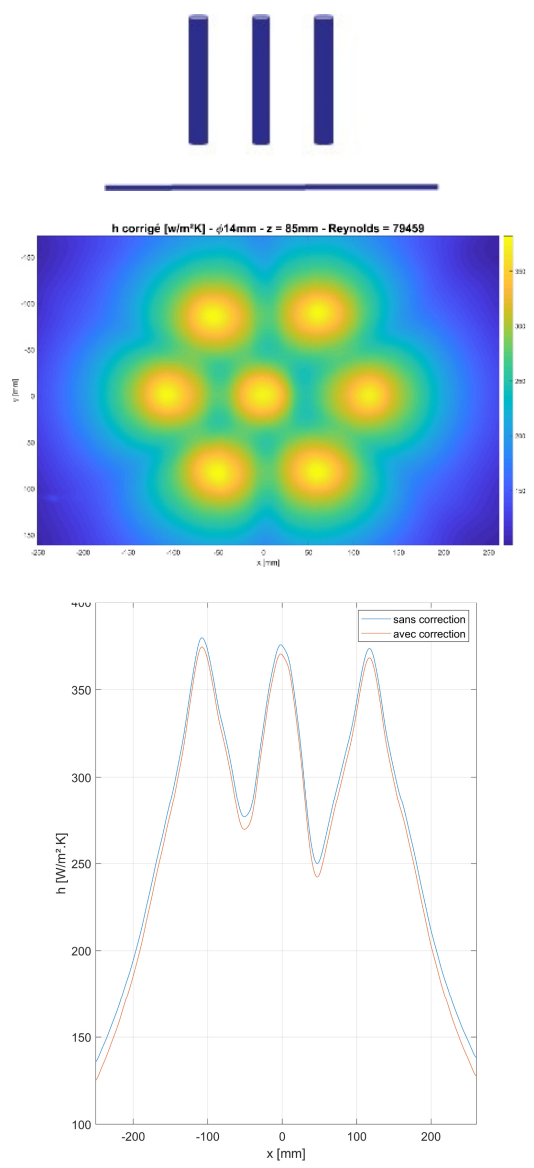

Inclined heated plate
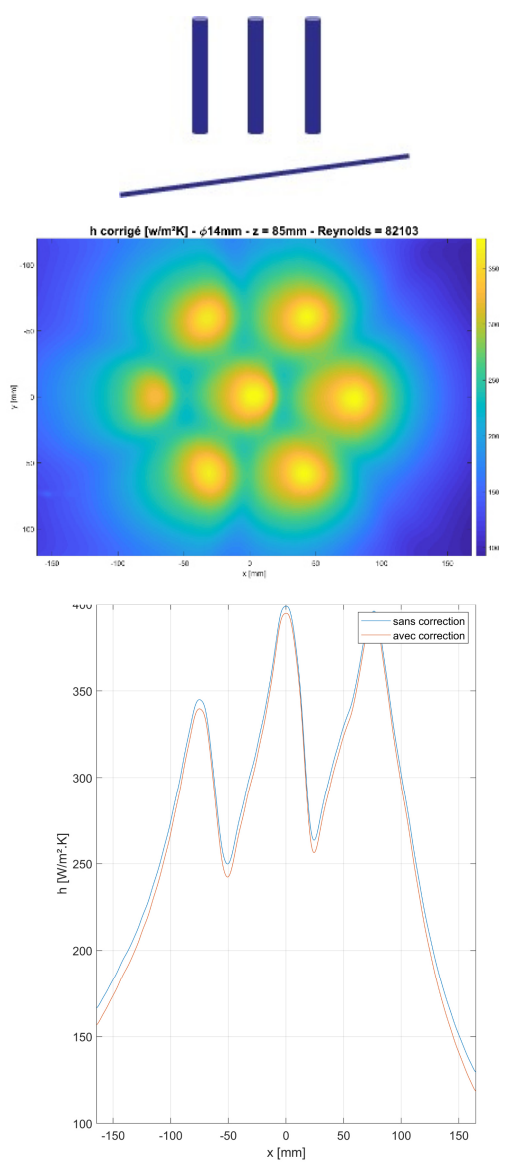

Figure 2 - typical results
Jets aligned with inclined heated plate


\section{REFERENCES}

[1] Martin H., "Heat and Mass Transfer between Impinging Gas Jets and Solid Surfaces". Advances in Heat Transfer, vol. 13, pp. 1-60, Academic Press, New York, 1977. 